\title{
Social Skills, Problem Behaviors and Classroom Management in Inclusive Preschool Settings*
}

\author{
Esra G. Karakaya ${ }^{1}$, Mumin Tufan ${ }^{2}$ \\ ${ }^{1}$ Pre-school Teacher, Kocaeli Provincial Directorate of National Education, Kocaeli, Turkey \\ ${ }^{2}$ Gazi Faculty of Education, Gazi University, Ankara, Turkey \\ Correspondence: Mumin Tufan, Gazi Faculty of Education, Gazi University, Emniyet Mahallesi / Bandırma Caddesi / \\ Bosna Binası No: 43206500 Beşevler/Yenimahalle/ Ankara, Turkey.
}

Received: March 5, 2018

doi:10.11114/jets.v6i5.3076
Accepted: April 7, $2018 \quad$ Online Published: April 9, 2018

URL: https://doi.org/10.11114/jets.v6i5.3076

\begin{abstract}
This study aimed to determine preschool teachers' classroom management skills and investigate the relationships between teachers' classroom management skills and inclusion students' social skills and problem behaviors. Relational screening model was used as the research method. Study group consisted of 42 pre-school teachers working in Kocaeli province and 42 inclusion students aged 4-7. Personal Information Form, Classroom Management Skills Inventory for Pre-school Teachers and Pre-School and Kindergarten Behavior Scale were used to collect data. Mann Whitney U and Kruskal Wallis methods were utilized to analyze the descriptive statistics. Results revealed that participating pre-school teachers had high level of perceived classroom management skills, teachers' classroom management skills did not differ based on inclusion students' age and gender but they significantly differed based on teachers' age, seniority, type of school they graduated from and staff status. Inclusion students were found to have moderate social skills and high level of problem behaviors. Children scores in social skills and problem behavior scales did not change based on age but they significantly differed based on gender. According to the results, no meaningful relationships existed between teachers' classroom management skills and inclusion students' social skills and problem behaviors.
\end{abstract}

Keywords: social skills, problem behavior, classroom management, inclusion students, pre-school

\section{Introduction}

Starting with their birth, children acquire information, skills and attitudes from their parents or caregivers related to various developmental areas. In later life, pre-primary educational institutions play a crucial role in providing children with different habits and life skills (Kandır, 2001). Preschool period is the most intensive period for children to acquire basic skills to adapt to environmental factors and society (Yaşar, 2008) and pre-schools are the first settings where children encounter social rules outside their families (Akduman, 2013).

Every child has a role to play in the society in which he lives. In order to be able to undertake these roles in a healthy manner, children must interact with others and acquire the ability to keep up with changing conditions. Just like healthy individuals, individuals with special needs have a number of roles in society. Individuals with special needs should be trained and supported in order to allow them to use their capacities to the fullest. Children with special needs should be given the opportunity to study in natural environments so that they are provided with equal opportunities in education. In this context, least restrictive educational environments are frequently used to meet the educational needs of children with special needs. Full-time or part-time training in regular classrooms for this purpose is called inclusive education (Kırcaali \& İftar, 2006).

The least restrictive learning environment principle allows students with special needs to share classroom environments with their typically developing peers and strengthen peer relationships (Mastropieri \& Scruggs, 2000). This way, children who complete their social development with their peers will have the opportunity to continue their lives in a

\footnotetext{
*This study was produced from the Master's Thesis titled "Investigation of the Relationship between Social Skills and Problem Behaviors of Inclusive Children and Classroom Management Skills of Preschool Teachers" completed at Gazi University.
} 
productive manner with acceptance throughout their lives starting from the classroom, the smallest educational unit (Kurcaali \& İftar, 2006).

Teachers are the individuals with whom children communicate the most during the pre-school period (Gülay-Ogelman \& Ersan, 2014). When the classroom environment is regarded as a small social unit, it will be apparent that children with special needs with healthy interclass interactions will be positively influenced. Teachers' attitudes towards children and the competences promoted by teachers have a high impact on children. One of the competences obtained at school is social skills. It is necessary for teachers involved in inclusive education to have sufficient knowledge about children's developmental characteristics and to display an appropriate and sensitive approach to these characteristics in the classroom (Allen \& Cowdery, 2005).

During pre-school education, children leave their home environment for the first time and take part in a social atmosphere with their age peers. Many social skills such as forming friendships, adapting to various environments and communicating are first introduced and developed here. Early social skills are closely related to later learning. For this reason pre-school education has a considerable impact on the development of social skills (Topaloğlu, 2013).

Effective classroom management is the first step to success in education (Sakarya, 2009). Teachers, regarded as one of the important elements in achieving the goals of the education program, should know children well, establish relationship based on love and trust and manage the classroom just like an orchestra conductor (Allen, 2010) with effective communication skills (Kandır, 2001).

Classroom management is a multi-dimensional concept that involves decision-making processes by taking individual differences into account. Planning and implementation of classroom activities, behavior management, organization of physical environment and educational materials can be regarded as the dimensions of classroom management (Şahin-Sak, 2015). Teachers' classroom management skills directly affect the quality of the training environment (Güven \& Cevher, 2005; Gülay-Ogelman \& Ersan, 2014. Using classroom management skills effectively is the most important role for the teacher in class. Therefore, it is necessary to identify desired behaviors so that measures should be taken to prevent possible problem behaviors. In classrooms where effective classroom management is available, the number of behaviors that are desired is relatively higher compared to undesired behaviors. Teachers with effective classroom management skills go beyond existing strategies and test new strategies (Uysal, Akbaba-Altun \& Akgül, 2010). These teachers are more productive in generating alternatives to possible challenges. In the same vein, it can be predicted that teachers who lack effective classroom management skills will have difficulty in implementing other skills efficiently and in helping students acquire desired behaviors (Terzi, 2002; Güven \& Cevher, 2005).

A positive classroom climate is crucial to achieve the intended success in education. Students feel more comfortable and secure in classrooms where classroom rules are identified and negative behaviors are minimized. Teachers need to be in charge of classroom management in order to prevent undesired behaviors in class. Effective classroom management is the first step of success in education (Sakarya, 2009).

Problem behaviors are a body of behaviors that impede the acceptance of the individual in the society, prevent adaptation of the individual to the environment and negatively affect the individual in all aspects (Aykır \& Tekinarslan, 2012). It is difficult to label a behavior as a problem behavior since problem behaviors may vary according to cultural characteristics of individuals who display them, persons who are affected by them and the society in which they are demonstrated (Aydin, 2000). Undesired behaviors may also vary according to age and gender of individuals and persistence, intensity and severity of the behavior (Aydoğmuş, 2010). Studies show that the origins of problem behaviors date back to early childhood years, problem behaviors become permanent over time if they do not receive appropriate responses and they may turn into greater learning difficulties in the future (Fischer, Rolf, Hasaz, \& Cummings, 1984).

Problem behaviors are closely related to inadequate social skills. Individuals with inadequate social skills resort to undesired behaviors to obtain what they want. This is more common in children with special needs compared to their peers with normal developmental patterns. Communication problems with peers caused by lack of social skills, not being well liked among peers, inadequate academic achievement and problem behaviors result in low social status and dislike towards school among inclusion students. Researchers found that inclusion students' academic achievement and social competencies are lower and their problem behaviors are three times higher than those of their typically developing peers (Aykır \& Tekinarslan, 2012; Yumuş \& Metin, 2015). In other words, there is a significant relationship between social skills, academic achievement and problem behaviors (Sucuoğlu \& Özokçu, 2005).

Due to physical and cognitive disabilities and lack of social competences, children with special needs have lower levels of communication with their peers and participation in group activities and they lack the ability to interact. Children with special needs experience behavioral disorders, they are isolated in the classroom environment and excluded by their peers (Yükselen \& Yaban, 2013) due to inadequate social skills. If early measures are not taken, problem behaviors such as truancy, involvement in criminal behaviors, loneliness, substance abuse, alcoholism, divorce and unemployment may be 
observed in the future (Özaydın, İftar \& Kaner, 2008; Yükselsin \& Berrakçay, 2010). For this reason, identifying inclusion students' social competences and problem behaviors will contribute to provision of developmental support (Yükselen \& Yaban, 2013).

Methods frequently used by teachers in pre-school education to overcome inclusion students' problem behaviors and increase their participation in activities usually involve getting detailed information from parents about their children's disability or learn methods that promote learning for students with specific type of disabilities with their individual efforts. Another method included extending the duration of classroom activities, being flexible in daily plans and striving to ensure full participation. In fact, classroom management skills play a crucial role in the education of inclusion students (Gülay-Ogelman \& Ersan, 2014) and there are no previous studies in Turkey that explored the relationship between preschool teachers' classroom management skills and inclusion students' social skills and problem behaviors. It is believed that this study, that set out to demonstrate the significance of classroom management in preschool education, will contribute to preschool teachers' practices. In this context; this study aimed to explore the relationship between preschool teachers' classroom management skill levels and inclusion students' social skills and problem behaviors. In this framework, answers were sought to the questions below:

1. What is the level of preschool teachers' classroom management skills based on their own perceptions?

2. Do preschool teachers' classroom management skills significantly differ based on demographic characteristics?

3. What is the level of inclusion students' social skills and problem behaviors?

4. Do inclusion students' social skills and problem behaviors significantly differ based on age and gender?

5. Is there a significant relationship between inclusion students' social skills and problem behaviors and preschool teachers' classroom management skills?

\section{Method}

\subsection{Model}

This research, which utilized relational screening model, set out to investigate the relationship between pre-school children's (aged 4-7) social skills and problem behaviors and pre-school teachers' classroom management skill levels.

\subsection{Participants}

Study participants were composed of 42 pre-school teachers employed in Kocaeli Province Izmit District and 42 inclusion students in these teachers' classrooms. In the study, 23,8\% $(\mathrm{n}=12)$ of the students were in $4-5$ age group and 76,2\% $(\mathrm{n}=30)$ were in 6-7 age group. In addition, 76,2\% of the inclusion students were $(\mathrm{n}=30)$ males and 23,8\% $(\mathrm{n}=12)$ were females.

In the study, $31 \%(\mathrm{n}=13)$ of the participating teachers were under 25 years, $38,0 \%(\mathrm{n}=16)$ were between $25-34,31,0 \%$ were $(n=13) 35$ or older. It was identified that $42,9 \%(n=18)$ of the teachers graduated from formal education institutions while $57,1 \%(\mathrm{n}=24)$ were graduates of distance education from Anadolu University. While the instructor and students are in the same setting in formal education, distance education is characterized by teaching when teachers and students are separated by physical distance (Verduin \& Clark, 1994).

It was also seen that $23,8 \%(\mathrm{n}=10)$ of the teachers had seniority of 1 year or under, $21,4 \%(\mathrm{n}=9)$ worked for $2-5$ years, 33,3\% $(\mathrm{n}=14)$ worked for $6-10$ years and $21,4 \%(\mathrm{n}=9)$ had a seniority 11 years or more.

\subsection{Data Collection Tools}

Personal Information Form, Classroom Management Skills Inventory for Pre-school Teachers and Pre-School and Kindergarten Behavior Scale (composed of Social Skills Scale and Problem Behaviors Scale) were used in this study as data collection tools. Scale questions were answered by participating teachers.

Personal Information Form prepared by the researcher includes questions to determine the following information for participating teachers: age, gender, seniority, staff status (whether they are on the permanent staff or paid by hour), the school teachers graduated from. The form also includes questions about pre-school students' ages and genders.

Classroom Management Skills Inventory for Pre-school Teachers, developed by Dinçer and Akgün (2015), was used to determine pre-school teachers' skill levels in classroom management. The 5-point Likert type scale is composed of 40 items that are scored by using "Strongly disagree", "Disagree", "Unsure", "Agree" and "Strongly agree". The scale includes two subscales: Professional Skills and Teacher-Child Interaction (Dinçer \& Akgün, 2015). Cronbach Alpha coefficient was calculated to determine the reliability of answers provided by teachers. Cronbach Alpha coefficient for items included in Professional Skills subscale was found to be 0.898 and Cronbach Alpha coefficient for items included in Teacher-Child Interaction was 0.888 while the Cronbach Alpha coefficient for the full scale was identified to be 0.929. 
Pre-School and Kindergarten Behavior Scale, developed by Merrell (1994) and translated by Fazlıoglu and Okyay (2011), was used to measure preschool children's problem behaviors and social skills. The scale is composed of two separate scales: Social Skills Scale and Problem Behaviors Scale. Items in the scale were rated by using "never", "rarely", "sometimes", and "often". The scale responses are coded as $0,1,2,3$ respectively. High scores from the general scale and from subscales indicate high level of social skills and problem behaviors while low scores point to low level of social skills and problem behaviors (Memetali, 2014).

Social Skills Scale (34 items) includes Social Cooperation, Social Independence and Social Interaction subscales. Cronbach alpha internal coefficient of consistence was found to be 0.749 for Social Cooperation, 0.603 for Social Interaction and 0.779 for Social Independence while it was 0.856 for the whole scale. These values show that scale items determine social skills of children reliably.

Problem Behaviors Scale (42 items) includes two subscales: Externalizing Problem Behaviors and Internalizing Problem Behaviors. Cronbach alpha coefficient was found to be 0.640 for Externalizing Problem Behaviors and 0.741 for Internalizing Problem Behaviors while it was 0.763 for the whole scale. Reliability coefficients show that scale items measure children's problem behaviors reliably.

\subsection{Data Collection Procedure}

Total number of pre-school inclusion students in Kocaeli Province central district and in separate educational institutions was obtained from Izmit Counseling and Research Center and required permits were received from Kocaeli Provincial Directorate of National Education. The 34 schools with inclusion students, identified in the framework of the study, were separately visited in a 2-month period in March and May of 2015-2016 academic year and the scales were implemented to 42 pre-school teachers.

\subsection{Data Analysis}

Firstly, descriptive statistics were undertaken based on children's and their teachers' scores from data collection tools. Later, Mann Whitney U test and Kruskal Wallis test were implemented for statistical calculations based on sub problems. Before analyses, hypothesis of normality was examined for the scores obtained from total scales and subscales.

\section{Results}

\subsection{What Is The Level of Preschool Teachers' Classroom Management Skills Based on Their Own Perceptions?}

Table 1. Descriptive statistics for pre-school teachers' classroom management skills scores

\begin{tabular}{lccccccc}
\hline Scale dimensions & Number of items & $\mathrm{N}$ & Lowest & Highest & $\overline{\mathrm{X}}$ & $\mathrm{SS}$ & Item means \\
\hline Professional Skills & 31 & 42 & 106,00 & 151,00 & 130,36 & 13,72 & 4,2 \\
Teacher-child interaction & 9 & 42 & 16,00 & 42,00 & 32,88 & 8,63 & 3,7 \\
Classroom management skills & 40 & 42 & 125,00 & 192,00 & 163,24 & 20,63 & 4,1 \\
\hline
\end{tabular}

Table 1 shows that participating pre-school teachers' classroom management skill scores differed between 125,00 and 192,00; their mean score was $163,24( \pm 20,63)$ and mean score for professional skills sub dimension was found to be $130,36( \pm 13,72)$ while the mean score for teacher-child interaction sub dimension was $32,88( \pm 8,63)$.

Table 2. Kruskal Wallis Test results for pre-school teachers' classroom management skills scale based on age

\begin{tabular}{|c|c|c|c|c|c|c|c|c|}
\hline Scale dimensions & Age & $\mathrm{N}$ & $\overline{\mathrm{x}}$ & SS & Mean rank & $\mathrm{X}^{2}$ & sd & $\mathrm{p}$ \\
\hline \multirow[t]{3}{*}{ Professional skills } & Under 25 & 13 & 117,77 & 8,62 & 10,38 & \multirow{3}{*}{16,304} & \multirow{3}{*}{2} & \multirow{3}{*}{$0,000^{*}$} \\
\hline & $25-34$ & 16 & 134,38 & 11,75 & 24,63 & & & \\
\hline & 35 and older & 13 & 138,00 & 11,83 & 28,77 & & & \\
\hline \multirow[t]{3}{*}{ Teacher-child interaction } & Under 25 & 13 & 26,77 & 7,10 & 13,96 & \multirow{3}{*}{11,786} & \multirow{3}{*}{2} & \multirow{3}{*}{$0,003^{*}$} \\
\hline & $25-34$ & 16 & 33,38 & 8,67 & 20,50 & & & \\
\hline & 35 and older & 13 & 38,38 & 6,04 & 30,27 & & & \\
\hline \multirow[t]{3}{*}{ Classroom management skills } & Under 25 & 13 & 144,54 & 13,35 & 10,23 & \multirow{3}{*}{17,486} & \multirow{3}{*}{2} & \multirow{3}{*}{$0,000^{*}$} \\
\hline & $25-34$ & 16 & 167,75 & 18,44 & 23,97 & & & \\
\hline & 35 and older & 13 & 176,38 & 16,03 & 29,73 & & & \\
\hline
\end{tabular}

Data presented here show that pre-school teachers' scores from classroom management skills scale significantly differed based on age in the whole scale $(X 2(2)=17,486 ; p>0,05)$, in professional skills sub dimension $(X 2(2)=16,304 ; p<0,05)$ and teacher-child interaction sub dimension $(\mathrm{X} 2(2)=11,786 ; \mathrm{p}<0,05)$. Kruskal Wallis test conducted to determine the source of this difference identified it to be in favor of teachers in 25-34 age range (between teachers who were under 25 and teachers between 25 and 34) and in favor of teachers who were 35 and older (between teachers who were under 25 and teachers who were 35 and older) in the whole scale and professional skills and teacher-child interaction sub dimensions. 
Table 3. Mann Whitney U Te st results for pre-school teachers' scores from classroom management skills scale based on type of school that teachers graduated from

\begin{tabular}{|c|c|c|c|c|c|c|c|c|c|}
\hline Scale dimensions & Type of school & $\mathrm{N}$ & $\overline{\mathrm{X}}$ & SS & Mean rank & Rank sum & $\mathrm{U}$ & $\mathrm{z}$ & $\mathrm{p}$ \\
\hline \multirow[t]{2}{*}{ Professional skills } & Formal education & 18 & 136,94 & 12,05 & 27,58 & 496,50 & \multirow[b]{2}{*}{106,500} & \multirow[b]{2}{*}{2,786} & \multirow[b]{2}{*}{$0,005^{*}$} \\
\hline & Distance education & 24 & 125,42 & 13,00 & 16,94 & 406,50 & & & \\
\hline \multirow[t]{2}{*}{ Teacher-child interaction } & Formal education & 18 & 36,50 & 6,79 & 26,58 & 478,50 & \multirow[b]{2}{*}{124,500} & \multirow[b]{2}{*}{2,338} & \multirow[b]{2}{*}{$0,019 *$} \\
\hline & Distance education & 24 & 30,17 & 8,98 & 17,69 & 424,50 & & & \\
\hline \multirow[t]{2}{*}{ Classroom management skills } & Formal education & 18 & 173,44 & 17,01 & 27,92 & 502,50 & \multirow[b]{2}{*}{100,500} & \multirow[b]{2}{*}{2,937} & \multirow[b]{2}{*}{$0,003 *$} \\
\hline & Distance education & 24 & 155,58 & 20,04 & 16,69 & 400,50 & & & \\
\hline
\end{tabular}

Data presented here show that pre-school teachers' scores from classroom management skills scale significantly differed based on type of school of graduation in the whole scale $(\mathrm{z}=2,937 ; \mathrm{p}<0,05)$, in professional skills sub dimension $(\mathrm{z}=2,786$; $p<0,05)$ and teacher-child interaction sub dimension $(z=2,338 ; p<0,05)$ in favor of teachers who graduated from formal education institutions.

Table 4. Kruskal Wallis Test results for pre-school teachers' classroom management skills scale based on seniority

\begin{tabular}{|c|c|c|c|c|c|c|c|c|}
\hline Scale dimensions & Seniority & $\mathrm{N}$ & $\overline{\mathrm{x}}$ & SS & Mean rank & $\mathrm{X}^{2}$ & sd & $\mathrm{p}$ \\
\hline \multirow[t]{4}{*}{ Professional skills } & 1 year and under & 10 & $116, \overline{90}$ & 5,78 & 10,00 & \multirow{4}{*}{20,782} & \multirow{4}{*}{3} & \multirow{4}{*}{$0,000 *$} \\
\hline & $2-5$ years & 9 & 124,33 & 12,03 & 15,00 & & & \\
\hline & $6-10$ years & 14 & 137,36 & 8,92 & 27,29 & & & \\
\hline & 11 year and higher & 9 & 140,44 & 13,64 & 31,78 & & & \\
\hline \multirow[t]{4}{*}{ Teacher-child interaction } & 1 year and under & 10 & 22,50 & 5,91 & 8,15 & \multirow{4}{*}{21,981} & \multirow{4}{*}{3} & \multirow{4}{*}{$0,000 *$} \\
\hline & $2-5$ years & 9 & 30,56 & 7,84 & 18,06 & & & \\
\hline & $6-10$ years & 14 & 37,29 & 5,41 & 26,25 & & & \\
\hline & 11 year and higher & 9 & 39,89 & 1,76 & 32,39 & & & \\
\hline \multirow[t]{4}{*}{ Classroom management skills } & 1 year and under & 10 & 139,40 & 6,93 & 8,25 & \multirow{4}{*}{25,174} & \multirow{4}{*}{3} & \multirow{4}{*}{$0,000 *$} \\
\hline & $2-5$ years & 9 & 154,89 & 18,44 & 15,50 & & & \\
\hline & $6-10$ years & 14 & 174,64 & 11,30 & 27,29 & & & \\
\hline & 11 year and higher & 9 & 180,33 & 15,29 & 33,22 & & & \\
\hline
\end{tabular}

Data presented here show that pre-school teachers' scores from classroom management skills scale significantly differed based on seniority in the whole scale $\left(X_{(3)}^{2}=25,174 ; p<0,05\right)$, in professional skills sub dimension $\left(X_{(3)}^{2}=20,782 ; p<0,05\right)$ and teacher-child interaction sub dimension $\left(\mathrm{X}_{(3)}^{2}=21,981 ; \mathrm{p}<0,05\right)$. Kruskal Wallis test conducted to determine the source of this difference identified that teachers with one year or less seniority and teachers with 2-5 years seniority had lower levels of classroom management skills in the whole scale and professional skills and teacher-child interaction sub dimensions.

Table 5. Mann Whitney U Test results for pre-school teachers' scores from classroom management skills scale based on staff status

\begin{tabular}{|c|c|c|c|c|c|c|c|c|c|}
\hline Scale dimensions & Staff Status & $\mathrm{N}$ & $\overline{\mathrm{X}}$ & SS & $\begin{array}{c}\text { Mean } \\
\text { rank }\end{array}$ & $\begin{array}{l}\text { Rank } \\
\text { sum }\end{array}$ & $\mathrm{U}$ & $\mathrm{Z}$ & $\mathrm{p}$ \\
\hline \multirow[t]{2}{*}{ Professional skills } & Permanent staff & 21 & 138,67 & 11,25 & 29,21 & 613,50 & \multirow[b]{2}{*}{58,500} & \multirow[b]{2}{*}{4,079} & \multirow[b]{2}{*}{$0,000^{*}$} \\
\hline & Paid by the hour & 21 & 122,05 & 10,70 & 13,79 & 289,50 & & & \\
\hline \multirow{2}{*}{$\begin{array}{l}\text { Teacher-child } \\
\text { interaction }\end{array}$} & Permanent staff & 21 & 38,10 & 4,65 & 28,12 & 590,50 & \multirow[b]{2}{*}{81,500} & \multirow[b]{2}{*}{3,516} & \multirow[b]{2}{*}{$0,000 *$} \\
\hline & Paid by the hour & 21 & 27,67 & 8,60 & 14,88 & 312,50 & & & \\
\hline \multirow{2}{*}{$\begin{array}{l}\text { Classroom } \\
\text { management skills }\end{array}$} & Permanent staff & 21 & 176,76 & 13,55 & 29,67 & 623,00 & \multirow[b]{2}{*}{49,000} & \multirow[b]{2}{*}{4,316} & \multirow[b]{2}{*}{$0,000 *$} \\
\hline & Paid by the hour & 21 & 149,71 & 17,45 & 13,33 & 280,00 & & & \\
\hline
\end{tabular}

Teachers' scores from classroom management skills scale were found to significantly differ based on staff status in the scale in general $(z=4,316 ; p<0,05)$, in professional skills sub dimension $(z=4,079 ; p<0,05)$ and teacher-child interaction sub dimension $(\mathrm{z}=3,516 ; \mathrm{p}<0,05)$.

\subsection{What Is The Level of Inclusion Students' Social Skills? Do Inclusion Students' Social Skills Significantly Differ Based} on Age and Gender?

Table 6. Descriptive statistics for inclusion students' social skills scale scores

\begin{tabular}{lccccccc}
\hline Scale dimensions & $\begin{array}{l}\text { Number } \\
\text { of items }\end{array}$ & $\mathrm{N}$ & Lowest & Highest & $\overline{\mathrm{X}}$ & $\mathrm{SS}$ & Item means \\
\hline Social Cooperation & 12 & 42 & 6,00 & 24,00 & 13,00 & 3,55 & 1,1 \\
\hline Social Interaction & 11 & 42 & 7,00 & 16,00 & 11,60 & 1,71 & 1,1 \\
\hline Social independence & 11 & 42 & 6,00 & 21,00 & 12,79 & 3,61 & 1,2 \\
\hline Social skill & 34 & 42 & 24,00 & 58,00 & 37,38 & 7,84 & 1,1 \\
\hline
\end{tabular}


Mean social skills score for inclusion students was $7,38( \pm 7,84)$ and this value was found to correspond to moderate level (Tekin, 2002). Sub dimensions were calculated as follows: mean Social Cooperation score: 13,00 $( \pm 3,55)$; mean Social Interaction: score 11,60 $( \pm 1,71)$ and mean Social Independence score: $12,79( \pm 3,61)$.

Table 7. Mann Whitney U Test results calculated for inclusion students' social skills scale scores based on age

\begin{tabular}{cccccccccc}
\hline \multicolumn{1}{c}{ Scale dimensions } & Age & $\mathrm{N}$ & $\overline{\mathrm{x}}$ & $\mathrm{SS}$ & Mean rank & Rank sum & $\mathrm{U}$ & $\mathrm{z}$ & $\mathrm{p}$ \\
\hline Social Cooperation & $4-5$ & 12 & 11,42 & 3,63 & 16,17 & 194,00 & & & \\
& $6-7$ & 30 & 13,63 & 3,37 & 23,63 & 709,00 & 116,000 & 1,798 & 0,077 \\
\hline Social Interaction & $4-5$ & 12 & 11,25 & 2,34 & 18,75 & 225,00 & & & \\
& $6-7$ & 30 & 11,73 & 1,41 & 22,60 & 678,00 & 147,000 & 0,943 & 0,371 \\
\hline Social independence & $4-5$ & 12 & 11,50 & 3,61 & 17,92 & 215,00 & & & \\
& $6-7$ & 30 & 13,30 & 3,54 & 22,93 & 688,00 & 137,000 & 1,205 & 0,240 \\
\hline \multirow{2}{*}{ Social skill } & $4-5$ & 12 & 34,17 & 8,00 & 16,42 & 197,00 & & & \\
\hline
\end{tabular}

$* \mathrm{p}<0,05$

No significant differences based on age were identified in inclusion students' social skills in general $(\mathrm{z}=1,702 ; \mathrm{p}>0,05)$. Also, no significant differences were identified based on age in Social Cooperation $(z=1,798 ; p>0,05)$, Social Interaction $(\mathrm{z}=0,943 ; \mathrm{p}>0,05)$ and Social Independence $(\mathrm{z}=1,205 ; \mathrm{p}>0,05)$ skills.

Table 8. Mann Whitney U Test results calculated for inclusion students' social skills scale scores based on gender

\begin{tabular}{|c|c|c|c|c|c|c|c|c|c|}
\hline Scale dimensions & Gender & $\mathrm{N}$ & $\overline{\mathrm{x}}$ & $\mathrm{SS}$ & Mean rank & Rank sum & $\mathrm{U}$ & $\mathrm{z}$ & $\mathrm{p}$ \\
\hline \multirow[t]{2}{*}{ Social Cooperation } & Male & 30 & $11, \overline{72}$ & 2,53 & 17,31 & 554,00 & \multirow[b]{2}{*}{26,000} & \multirow[b]{2}{*}{3,994} & \multirow[b]{2}{*}{$0,000 *$} \\
\hline & Female & 12 & 17,10 & 3,28 & 34,90 & 349,00 & & & \\
\hline \multirow[t]{2}{*}{ Social Interaction } & Male & 30 & 11,16 & 1,59 & 18,09 & 579,00 & \multirow[b]{2}{*}{51,000} & \multirow[b]{2}{*}{3,305} & \multirow[b]{2}{*}{$0,001 *$} \\
\hline & Female & 12 & 13,00 & 1,33 & 32,40 & 324,00 & & & \\
\hline \multirow[t]{2}{*}{ Social independence } & Male & 30 & 11,47 & 2,92 & 17,28 & 553,00 & \multirow[b]{2}{*}{25,000} & \multirow[b]{2}{*}{4,013} & \multirow[b]{2}{*}{$0,000 *$} \\
\hline & Female & 12 & 17,00 & 2,05 & 35,00 & 350,00 & & & \\
\hline \multirow[b]{2}{*}{ Social skill } & Male & 30 & 34,34 & 5,83 & 16,97 & 543,00 & \multirow[b]{2}{*}{15,000} & \multirow[b]{2}{*}{4,292} & \multirow[b]{2}{*}{$0,000 *$} \\
\hline & Female & 12 & 47,10 & 5,00 & 36,00 & 360,00 & & & \\
\hline
\end{tabular}

Compared to male students $(34,34 \pm 5,83)$; female students $(47,10 \pm 5,00)$ were found to obtain significantly higher scores based on mean rank and total scores for social skills $(z=4,292 ; p<0,05)$. Similarly, inclusion students' social skills significantly differed in favor of female students in Social Cooperation $(z=3,994 ; p<0,05)$, Social Interaction $(z=3,305$; $\mathrm{p}<0,05)$ and Social Independence $(\mathrm{z}=4,013 ; \mathrm{p}<0,05)$ sub dimensions.

3.3 What Is the Level of Inclusion Students' Problem Behaviors? Do Inclusion Students' Problem Behaviors Significantly Differ Based on Age and Gender?

Table 9.Descriptive statistics for inclusion students' problem behaviors scale scores

\begin{tabular}{lccccccc}
\hline Scale dimensions & $\begin{array}{l}\text { Number } \\
\text { of items }\end{array}$ & $\mathrm{N}$ & Lowest & Highest & $\overline{\mathrm{X}}$ & SS & Item means \\
\hline Externalizing problem & 27 & 42 & 47,00 & 74,00 & 66,88 & 6,14 & 2,5 \\
\hline Internalizing problem & 15 & 42 & 33,00 & 41,00 & 37,45 & 2,11 & 2,5 \\
\hline Problem behavior & 42 & 42 & 81,00 & 113,00 & 104,33 & 6,94 & 2,5 \\
\hline
\end{tabular}

Mean problem behavior score for inclusion students was 104,33 $( \pm 6,94)$ which was identified to be higher in the scale in general and in sub scales (Tekin, 2002). Examination of sub scales provides the mean score for externalizing problem behavior as $66,88( \pm 6,14)$ and the mean score for internalizing problem behavior as $37,45( \pm 2,11)$.

Table 10. Mann Whitney U Test results calculated for inclusion students' problem behavior scale scores based on age

\begin{tabular}{|c|c|c|c|c|c|c|c|c|c|}
\hline Scale dimensions & Age & $\mathrm{N}$ & $\mathrm{x}$ & SS & Mean rank & Rank sum & $\mathrm{U}$ & $\mathrm{Z}$ & $\mathrm{p}$ \\
\hline \multirow[t]{2}{*}{ Externalizing problem } & $4-5$ & 12 & 67,25 & 5,86 & 22,46 & 269,50 & \multirow[b]{2}{*}{168,500} & \multirow[b]{2}{*}{0,322} & \multirow[b]{2}{*}{0,752} \\
\hline & $6-7$ & 30 & 66,73 & 6,34 & 21,12 & 633,50 & & & \\
\hline \multirow[t]{2}{*}{ Internalizing problem } & $4-5$ & 12 & 38,00 & 2,04 & 25,13 & 301,50 & \multirow[b]{2}{*}{136,500} & \multirow[b]{2}{*}{1,226} & \multirow[b]{2}{*}{0,229} \\
\hline & $6-7$ & 30 & 37,23 & 2,13 & 20,05 & 601,50 & & & \\
\hline \multirow[t]{2}{*}{ Problem behavior } & $4-5$ & 12 & 105,25 & 6,41 & 23,29 & 279,50 & \multirow[b]{2}{*}{158,500} & \multirow[b]{2}{*}{0,601} & \multirow[b]{2}{*}{0,554} \\
\hline & $6-7$ & 30 & 103,97 & 7,21 & 20,78 & 623,50 & & & \\
\hline
\end{tabular}

*p $<0,05$

Data presented here show no significant differences for inclusion students' problem behaviors based on age ( $\mathrm{z}=0,601$; $\mathrm{p}>0,05)$. Similarly, no significant differences were determined for externalizing problem behavior $(\mathrm{z}=0,322 ; \mathrm{p}>0,05)$ and Internalizing problem behavior $(\mathrm{z}=1,226 ; \mathrm{p}>0,05)$ subscales as well. 
Table 11. Mann Whitney U Test results calculated for inclusion students' problem behavior scale scores based on gender

\begin{tabular}{|c|c|c|c|c|c|c|c|c|c|}
\hline Scale dimensions & Gender & $\mathrm{N}$ & $\overline{\mathrm{x}}$ & SS & Mean rank & Rank sum & $\mathrm{U}$ & $\mathrm{Z}$ & $\mathrm{p}$ \\
\hline \multirow[t]{2}{*}{ Externalizing problem } & Male & 30 & 69,31 & 4,00 & 26,09 & 835,00 & & & \\
\hline & Female & 12 & 59,10 & 5,32 & 6,80 & 68,00 & 13,000 & 4,359 & $0,000 *$ \\
\hline \multirow[t]{2}{*}{ Internalizing problem } & Male & 30 & 37,44 & 2,11 & 21,67 & 693,50 & & & \\
\hline & Female & 12 & 37,50 & 2,22 & 20,95 & 209,50 & 154,500 & 0,164 & 0,873 \\
\hline \multirow[t]{2}{*}{ Problem behavior } & Male & 30 & 106,75 & 5,16 & 25,92 & 829,50 & & & \\
\hline & Female & 12 & 96,60 & 6,35 & 7,35 & 73,50 & 18,500 & 4,195 & $0,000^{*}$ \\
\hline
\end{tabular}

Based on the data presented in Table 11, the level of displaying problem behaviors significantly differed among inclusion students based on gender $(z=4,195 ; \mathrm{p}<0,05)$. Male students' level of displaying problem behaviors $(106,75 \pm 5,16)$ was identified to be higher compared to that of female students $(96,60 \pm 6,35)$ considering the mean rank and total scores in the scale as a whole. Similarly, sub scales also show that externalizing problem behaviors $(z=4,359 ; p<0,05)$ and internalizing problem behaviors $(\mathrm{z}=0,164 ; \mathrm{p}>0,05)$ significantly differed in favor of male students.

3.4 Is There a Significant Relationship Between Inclusion Students' Social Skills and Level of Displaying Problem Behaviors and Preschool Teachers' Classroom Management Skills?

Table 12. Relationships between inclusions students' social skills and pre-school teachers' classroom management skills

\begin{tabular}{|c|c|c|c|c|}
\hline Scale dimensions & Values & Professional skills & Teacher-child interaction & Classroom management \\
\hline \multirow{3}{*}{ Social Cooperation } & $\mathrm{r}$ &, 186 &, 108 &, 169 \\
\hline & $\mathrm{p}$ & 237 & ,495 & ,284 \\
\hline & $\mathrm{N}$ & 42 & 42 & 42 \\
\hline \multirow[t]{3}{*}{ Social Interaction } & $\mathrm{r}$ &,- 109 &,- 198 &,- 155 \\
\hline & $\mathrm{p}$ &, 492 & ,208 &, 326 \\
\hline & $\mathrm{N}$ & 42 & 42 & 42 \\
\hline \multirow[t]{3}{*}{ Social Independence } & $\mathrm{r}$ & ,207 & 106 & 182 \\
\hline & $\mathrm{p}$ &, 189 & ,506 & 249 \\
\hline & $\mathrm{N}$ & 42 & 42 & 42 \\
\hline \multirow[t]{3}{*}{ Social Skill } & $\mathrm{r}$ & , 156 &, 054 &, 126 \\
\hline & $\mathrm{p}$ & ,324 & ,732 & ,425 \\
\hline & $\mathrm{N}$ & 42 & 42 & 42 \\
\hline
\end{tabular}

$* * \mathrm{p}<0,01 ; * \mathrm{p}<0,05$

Data included on Table 12 demonstrate no significant relationships between inclusion students' social skills and their teachers' professional skills $(r=0,156 ; p>0,05)$, teacher-child interaction skills $(r=0,054 ; p>0,05)$ and classroom management skills $(r=0,126 ; p>0,05)$.

Table 13. Relationships between inclusion students' problem behaviors and pre-school teachers' classroom management skills

\begin{tabular}{|c|c|c|c|c|}
\hline Scale dimensions & Values & Professional skills & Teacher-child interaction & Classroom management \\
\hline \multirow[t]{3}{*}{ Externalizing problem } & $\mathrm{r}$ &,- 236 &,- 296 &,- 281 \\
\hline & $p$ & ,133 & ,057 & ,072 \\
\hline & $\mathrm{N}$ & 42 & 42 & 42 \\
\hline \multirow[t]{3}{*}{ Internalizing problem } & $\mathrm{r}$ &,- 130 &,- 020 &,- 095 \\
\hline & $\mathrm{p}$ & ,410 & ,901 & ,549 \\
\hline & $\mathrm{N}$ & 42 & 42 & 42 \\
\hline \multirow{3}{*}{ Problem behavior } & $\mathrm{r}$ & $\begin{array}{l}-248 \\
\end{array}$ & $\begin{array}{l}-268 \\
\end{array}$ &,- 277 \\
\hline & $\mathrm{p}$ & ,113 & ,086 &, 075 \\
\hline & $\mathrm{N}$ & 42 & 42 & 42 \\
\hline
\end{tabular}

$* * \mathrm{p}<0,01 ; * \mathrm{p}<0,05$

No significant relationships were identified between participating pre-school students' problem behaviors and their teachers' professional skills $(r=-0,248 ; p>0,05)$, teacher-child interaction skills $(r=-0,268 ; p>0,05)$ and classroom management skills $(r=-0,277 ; p>0,05)$.

\section{Discussion}

Mean scores that teachers obtained from Classroom Management Skills Inventory for Pre-school Teachers in general and the mean scores obtained from scale sub dimensions in the study presented that teachers had high level of perceptions related to their classroom management skills. In other words, participating teachers' perceptions related to their classroom management skills were rather positive. It was also identified that the score obtained from professional skills 
sub dimension was higher than the score obtained from teacher-child interaction sub dimension. This finding may show that while teachers worked together with inclusion students, they did not interact with them sufficiently.

According to studies that focus on classroom management; Adıgüzel \& Cülha (2016) reported that teachers perceived their classroom management skills as sufficient or high and they though they "mostly" carried out these skills and Koçoğlu (2013) found that teachers performed "a bit higher than the moderate level" in classroom management mean scores. The results of this study support such previous findings.

However, some research findings emphasize the fact that teachers have limited or low level classroom management skills. Gezgin (2009) reported that pre-school teachers or pre-school teacher candidates have information about classroom management strategies in general but they do not use some strategies almost at all while Güner (2011) found that teachers have limited classroom management knowledge.

Regarding the relationship between teachers' classroom management skills and age; it was identified that classroom management skills were significantly lower in teachers younger than 25 and in teachers between 25 and 34 years of age compared to teachers who are 35 years or older. In other words, teachers' perceptions regarding their classroom management skills increased in the scale in general and in scale sub dimensions along with increases in age. This finding is thought to be associated with increased professional experience by age, personal development and experience.

Durğun (2010) found that classroom management skills of teachers who were 51 years or older were significantly higher than those of other groups and this finding supports the findings of the current study. However, contrary to the findings of this study, Güner (2011) found that classroom management knowledge of teachers who were 45 or younger were higher than those who were 46 or older.

Teachers' classroom management skill levels significantly differed based on the type of school they graduated from. Compared to teachers who graduated from distance education; teachers who graduated from formal educational institutions obtained significantly higher scores in classroom management skills including sub scales. This finding indicates that compared to teachers who graduated from distance education institutions; teachers who graduated from universities that provide formal education had more positive views on classroom management. It is believed that teachers' positive views on classroom management may be affected by the fact that formal education is conducted via one-on-one contact and supports face to face interaction and one-on-one and active learning. This finding is supported by similar studies. Dinçer and Akgün (2015) reported that classroom management skills of teachers who graduated from formal faculties of education were higher compared to those of teachers who graduated from distance education Faculty of Education. According to Kale \& Baburşah (2017), classroom management inclinations of teachers do not significantly differ based on the type program teachers graduate from.

Teachers' classroom management skills differed based on seniority. Compared to teachers with higher seniority, classroom management skills of teachers with professional experience of one year or less and teachers with 2-5 years of experience were lower in professional skills and teacher-child interaction sub scales. This result indicates that professional experience positively affects teachers' classroom management performance. As teachers' professional experiences increase, their classroom management performances also improve. It can be claimed that experience is an important element in teaching profession due to its direct influence on classroom management. Similarly, Yumuş (2013) found that teachers with 15 years or more experience were better at coping with undesired behaviors in class compared to their colleagues with less experience. In his study "Classroom Management: Beliefs of Preservice Teachers and Classroom Teachers Concerning Classroom Management Styles" Laut (1999) explored the impact of seniority on teachers' classroom management practices. According to the results of the study, compared to the other group; teachers with at least 3 years of professional experience adopted interventionist approach at a lesser degree. Martin and Shoho (2000) and Martin, Yin and Mayall (2006) examined the relationship between teachers' classroom management attitudes and their professional seniority. Both studies demonstrated that compared to novice teachers, teachers with many years of experience utilized a more interventionist approach towards children in classroom management. However, Yüksel (2013) reported that teachers' classroom management skills do not differ according to professional seniority with the exception of "motivation" sub scale.

Teachers' classroom management skills differed based on staff status. Professional skills and teacher-child interaction skills of the teachers in permanent staff were found to be significantly higher than those of teachers who were paid by the hour. According to this finding, staff status led to significant differences in perceptions regarding classroom; this may be related to the fact that compared to teachers that are paid by the hour, teachers in permanent staff are more likely to develop a sense of belonging to their schools. In addition, this sense of belonging may lead teachers to embrace their schools and classrooms in such a degree to motivate increases in the use of better classroom management skills. Similarly, Dinçer and Akgün (2015) stated that being on permanent staff had a significant effect on perceived classroom management skills. On the other hand, Adıgüzel (2016) expressed that being on permanent staff had no significant effect 
on perceived classroom management skills and teachers who were on permanent staff or who were paid by the hour obtained almost the same scores in classroom management skills scale.

When inclusion students' scores from Social Skills Scale were assessed in general based on teacher responses, it was found that inclusion students had moderate level social skills. Similarly, Poyraz-Tüy (1999) reported that scores obtained by special needs students from social independence and social cooperation sub scales of social skills scale did not differ significantly from the scores obtained by their peers with normal developmental patterns.

Inclusion students' scores on social skills scale did not display significant differences based on age. In other words, inclusion students who were 4-5 or 6-7 had similar social cooperation, social interaction and social independence skills and age did not play a determinant role in social skills. Different from the findings of this study, Merrell (1994) and Poyraz-Tüy (1999) found that social skills develop by age. Aydın \& Şen-Sönmez (2014) and Aydın \& Sönmez (2014) reported that while age factor does not directly increase social skills, it may be related to them.

Inclusion students' scores from social skills scale in relation to gender show that scores obtained by female students were higher than those obtained by male students in social cooperation, social interaction and social independence. According to this finding, compared to age, gender plays a more determinant role on social skills. It was found in this study that while 4 and 7 year-old inclusion students had similar social skills, 4 year-old male and female students had rather different social skills. Aykır (2010) reported that male students had less social skills compared to female students and this finding supports the findings of the current study. However, Aydın \& Şen-Sönmez (2014) stated that there were no significant differences between social skills of mentally disabled children and gender.

Based on the scores obtained by inclusion students in Problem Behaviors Scale and its sub scale dimensions; inclusion students were identified to have high levels of internalizing and externalizing problem behaviors. In the scope of the study, inclusion students' social skills were found to be at moderate level while their problem behaviors were rather high. This finding can be interpreted to mean that there is an inverse relationship between social skills and problem behaviors. This finding points to high tendencies of displaying problem behaviors by inclusion students and it is similar to the results of many studies in literature. Poyraz-Tüy (1999) reported that hearing impaired students that attended special education institutions displayed more internalizing problem behaviors compared to their peers with normal developmental patterns. Gök (2013) included the views of teachers who had inclusion students in their classrooms. According to these teachers, inclusion students experienced adaptation problems from time to time and displayed aggressive behaviors.

Scores obtained by inclusion students from Problem Behaviors Scale did not differ based on age. In other words, inclusion students who were 4-5 or 6-7 years of age had similar tendencies to display internalizing or externalizing problem behaviors. The finding by Sucuoğlu and Özokçu (2005) and Aydın \& Şen-Sönmez (2014) that there were no relationships between disabled children's problem behavior levels and their age support the findings of this study. On the contrary, in their study, Alisinanoğlu and Kesicioğlu (2010) found that children differed based on age in the inattentiveness and hyper activity sub dimensions of problem behavior scale.

Scores obtained by inclusion students in Problem Behaviors Scale did not significantly differ based on gender. It was found that male students had higher tendencies to display externalizing problem behaviors compared to female students. This result implies that inclusion students' gender is more dominant in determining problem behaviors compared to their age. In other words, a young female inclusion student in the study is less likely to display negative behaviors compared to an older male inclusion student. In their research, Sucuoğlu and Özokçu (2005) reported that scores obtained from problem behavior scale were in favor of male students; in other words, male students displayed more problem behaviors compared to female students. Yoşumaz (2013) stated that inclusion students' problem behaviors did not significantly differ according to gender.

In their study titled "Teacher Classroom Management Practices: Effects on Disruptive or Aggressive Student Behavior"; Oliver, Wehby, and Reschly (2011) investigated the relationship between teachers' classroom management practices and their students' problem behaviors. Results pointed to a meaningful relationship between teacher's classroom management skills and in-class problem behaviors. Contrary to this finding, the study results point to no significant relationships between teachers' scores in classroom management skills scale and students' scores in social skills and problem behavior scales. Therefore, it was determined that inclusion students' social skills and problem behaviors were not significantly related to teachers' classroom management skills. This result may be related to unbalanced distribution of the study group in terms of gender characteristics (such as the fact that all teachers were females, large number of male students compared to female students etc.), setting of the study and the societal structure, characteristics of the scale used in the study and limited number of participants.

Overall assessment of the main findings of the study shows that classroom management skills differentiated according to teacher characteristics, a meaningful difference existed between students' gender and their social skills and problem 
behaviors however there was no significant difference between teachers' classroom management skills and their students' social skills and problem behaviors

This study has some limitations. It was conducted with a total of 42 pre-school teachers and 42 inclusion students attending pre-schools based on primary schools affiliated with Ministry of National Education, independent and private kindergartens located in the central district of a province in Turkey. The majority of participating inclusion students were male whereas all teachers were females. Therefore, the results of this study are not generalizable to all Turkish contexts.

Some suggestions based on the results of this study are as follows: A large majority of inclusion students were found to obtain sufficient scores from Problem Behaviors Scale. Therefore, sources of the problem behaviors, which are listed in the scale, found to be frequently repeated can be investigated. Training programs can be organized by experts to minimize these behaviors in the classroom. Teachers can be provided with in-service training programs on the strategies that can be utilized to minimize problem behaviors in class. The study group was very limited in this study due to its focus on inclusion students. Future studies may be extended to teachers who do not have inclusion students in their classrooms to have comparative analysis of classroom management skills employed by these two teacher groups. Teachers' classroom management skills may be investigated based on various factors including their attendance in in-service training activities, having inclusion students in their classrooms, number of students in their classrooms, their marital status and whether they have children. Factors affecting children's social skills and problem behaviors can be explored by including additional factors such as disability status, number of siblings and duration of school attendance.

\section{References}

Adiguzel, A., \& Culha A. (2016). Comparison of classroom management skills of teachers graduated from education faculties and others. Asian Journal of Instruction, 4(1), 17-33.

Adiguzel, I. (2016). The relations between pre-school teachers' classroom management skills and burnout level (Unpublished master's thesis). Recep Tayyip Erdoğan University, Rize, Turkey.

Akduman, G. G. (2013). Definition and importance of pre-school education. In G. Uyanık Balat (Ed.), Introduction to pre-school education (pp. 1-18). Ankara: Pegem A Publications.

Alisinanoglu, F., \& Kesicioglu, O. S. (2010). The research on the behavioral problems of preschoolers in terms of various variables (An example of Giresun province). Journal of Theoretical Educational Science, 3(1), 93-110.

Allen, K. E., \& Cowdery, G. E. (2005). The exceptional child: Inclusion in early childhood education. Clifton Park, NY: Thomson Delmar Learning.

Allen, K. P. (2010). Classroom management, bullying, and teacher practices. The Professional Educator, 34(1), 1-15.

Aydin, A. (2000). Classroom management. Ankara: Anı Publishing.

Aydın, A., \& Sen-Sonmez, A. (2014). Relationship between social skills and articulation levels of children with mental disability. The Journal of Academic Social Science, 1, 305-320. https://doi.org/10.16992/ASOS.92

Aydın. A., \& Sönmez, O. I. (2014). The effect of rearing attitudes of mothers having intellectually disabled children to the social skills of their children. YYU Journal Of Education Faculty, XI(I), 149-168.

Aydogmus, K. (2010). Parent school. Istanbul: Remzi Bookstore.

Aykir, T. (2010). The comparison of preschool children with mental retardation in terms of social skills and problem behavior (Unpublished master's thesis). Abant Izzet Baysal University, Bolu, Turkey. A

Aykir, T., \& Tekinarslan, I. C. (2012). A comparison of social skills and problem behaviors of preschool children with and without mental disability. Kastamonu Education Journal, 20(2), 627-648.

Denizel-Guven, E., \& Cevher, N. (2005). The level of preschool teachers' classroom management skills and its relations with different variables. Pamukkale University Journal of Education, 18(1), 1-22.

Dincer, C., \& Akgun, E. (2015). Developing a classroom management skills inventory for preschool teachers and the correlation of preschool teachers' classroom management skills with different variables. Education and Science, 40(177), 187-201. https://doi.org/10.15390/EB.2015.2

Durgun, B. (2010). Depending on various variables, examining classroom management skills of teachers who have students that need special training (An example of Sancaktepe district) (Unpublished master's thesis). Yeditepe University, İstanbul, Turkey.

Fazlioglu, Y., Okyay, L., \& Ilgaz, G. (2011). Study validity and reliability to preschool and kindergarten behavior scales-PKBS-2. Trakya University Journal of Social Science, 13(1), 253-266.

Fischer, M., Rolf, J. E., Hasazi, J. E., \& Cummings, L. (1984). Follow-up of a preschool epidemiological sample: 
Cross-age continuities and predictions of later ad- patterns and children's behavior problems from injustment with internalizing and externalizing dimensions of behavior. Child Development, 55(1), 137-150. https://doi.org/10.2307/1129840

Gezgin, N. (2009). The classroom management strategies that preschool education teachers use (Unpublished master's thesis). Uludağ University, Bursa, Turkey.

Gok, R. (2013). The problems that primary school teachers, who have also students with special needs in their classrooms, experience in classroom management and the strategies they use to overcome them (Unpublished master's thesis). Akdeniz University, Antalya, Turkey.

Gulay-Ogelman, H., \& Ersan, C. (2014). The effect classroom management strategies for preschool teachers has on peer relationships in children. Bartin University Journal of Faculty of Education, 3(2), 63-84. https://doi.org/10.14686/BUEFAD.201428172

Guner, N. (2011). Examining the level of classroom management knowledge of teachers in inclusive classrooms. Kastamonu Ë̆itim Dergisi, 19(3), 691-708.

Kale, M., \& Babursah D. (2017). Determination of classroom teachers' approach to classroom management. Karaelmas Journal of Educational Sciences, 5, 75-88.

Kandir, A. (2001). Place and importance of preschool education institutions in child development. Journal of National Education, 151, 51-65. http://yayim.meb.gov.tr/dergiler/151/kandir.htm

Kircaali-Iftar, G. (2006). Scientific Research Methods. Lecture Notes. Anadolu University.

Kocoglu, A. M. (2013). Multidimensional research on classroom management skills of form teachers in primary schools (Sancaktepe sample) (Unpublished master's thesis). Yeditepe University, Istanbul, Turkey.

Laut, J. (1999). Classroom management: beliefs of preservice teachers and classroom teachers concerning classroom management styles. Paper presented at the Fall Teachers Education Conference. Charleston University, South Carolina. Abstract retrieved from https://eric.ed.gov/?id=ED445815

Martin, N. K., \& Shoho, A. R (2000, January). Teacher experience, training \& age: The influence of teacher characteristics on classroom management style. Paper presented at the Annual Meeting of the Southwest Educational Research Association, Texas. Abstract retrieved from https://eric.ed.gov/?id=ED440963

Martin, N. K., Yin, Z., \& Mayall, H. (2006, February). Classroom management training, teaching experience and gender: Do these variables impact teachers' attitudes and beliefs toward classroom management style. Paper presented at the Annual Conference of the Southwest Educational Research Association, Texas. Abstract retrieved from https://eric.ed.gov/?id=ED494050

Mastropieri, M., \& Scruggs, T. (2000). The inclusive classroom strategies for effective instruction. Upper Saddle River, NJ: Merrill/Prentice Hall.

Memetali, S. (2014). Validity and reliability study of the preschool and kindergarten behavior scale in the case of western Thrace (Unpublished master's thesis). Trakya University, Edirne, Turkey.

Merrell, K. W. (1994). Test manual of preschool and kindergarten behavior scales. Brandon: Clinical Psychology.

Oliver, R. M., Wehby, J. H., \& Reschly, D. J. (2011). Teacher Classroom Management Practices: Effects on Disruptive or Aggressive Student Behavior. Paper presented at the Society for Research on Educational Effectiveness Conference, Netherlands. https://doi.org/10.4073/csr.2011.4

Ozaydın, L., Tekin-Iftar, E., \& Kaner, S. (2008). The effects of a friendship development program on the social interactions of the preschool children with special needs. Ankara University Faculty of Educational Sciences Journal of Special Education, 9(1), 15-32.

Poyraz-Tuy, S. (1999). Being comparated with point of the social skill and problem behaviors of hearing impaired and hearing children whose ages are between 3-6 (Unpublished master's thesis). Ankara University, Ankara, Turkey.

Sahin-Sak, I. T. (2015). Pre-service preschool teachers' self-efficacy beliefs about classroom management. Sakarya University Faculty of Education Journal, 29, 101-120.

Sakarya, A. R. (2009). Elementary school students' awareness level of their problem behaviors in the classroom (Unpublished master's thesis). Yeditepe University, İstanbul, Turkey.

Sucuoglu, B., \& Ozokcu, O. (2005). Evaluation of social skills of mainstreaming students. Ankara University Faculty of Educational Sciences Journal of Special Education, 6(1), 41-57. 
Tekin, H. (2002). Assessment and evaluation in education. Ankara: Yarg1 Publishing.

Terzi, A. R. (2002). Effective teacher behaviors in terms of classroom management. Journal of National Education, 1(1), 155-156.

Topaloglu, A. (2013). The influence of activity based social skills education on children's peer relationships (Unpublished doctoral dissertation). Selçuk University, Konya, Turkey.

Uysal, H., Akbaba-Altun, S., \& Akgun, E. (2010). The strategies preschool teachers use when confronted with children's undesired behaviors. Illkögretim Online, 9(3), 971-979.

Verduin, J. R., \& Clark, T. A. (1994). Distance education: The foundations of effective practice. (Trans. İlknur Maviş) Eskişehir: Anadolu Üniversity Press.

Yasar, S. (2008). Gaining basic habits and social behaviors in preschool education. In M. Şişman \& S. Turan (Ed.), Introduction to preschool education (pp. 10). Eskişehir: Anadolu University Press.

Yosumaz, K. (2013). Comparison of mentally retarded children and problematic behaviors of children with normal development (60-72 months) going to preschool institutions according to different variables (Unpublished master's thesis). Dumlupınar University, Kütahya, Turkey.

Yuksel, A. (2013). The assessment on classroom management skills of primary school teachers: A case study of Afyonkarahisar (Unpublished doctoral dissertation). Gazi University, Ankara, Turkey.

Yukselen, A., \& Yaban, H. (2013). Social competence of integrated children with mild intellectual disability. Hacettepe University Journal of Education, 28(3), 457-471.

Yukselsin, I. Y., \& Berrakcay, O. (2010). The impacts of 'interactive rhythmic repetition exercise' as a model of musical therapy on reduction the problem behaviors of children with autistic spectrum disorders. The Journal of International Social Research, 3(10), 661-673.

Yumus, M. (2013). Research on opinions of preschool teachers about behavioral problems of 36-72 month-old children and determining coping strategies (Unpublished master's thesis). Hacettepe University, Ankara, Turkey.

Yumus, M., \& Metin, N. (2015). Causes of behavior problems in inclusion classes and managing strategies. Hacettepe University Faculty of Health Sciences Journal, 1(2), 474-482.

\section{Copyrights}

Copyright for this article is retained by the author(s), with first publication rights granted to the journal.

This is an open-access article distributed under the terms and conditions of the Creative Commons Attribution license which permits unrestricted use, distribution, and reproduction in any medium, provided the original work is properly cited. 